\title{
Contingency Ranking in Modern Power System by Exact and Precise Method
}

\author{
Udaykumar $^{1}$, H.R.Sudarshana Reddy ${ }^{2}$ \\ P.G. Student, E.E.E Department, UBDT College of Engineering, Davangere, Karnataka, India ${ }^{1}$ \\ Professor, E.E.E Department, UBDT College of Engineering, Davangere, Karnataka, India ${ }^{2}$
}

\begin{abstract}
One of the important aspects of modern power system security assessment is the consideration of any contingencies arises due to unplanned or planned line outages leading to system overloads or abnormal system voltages. Several methods have been developed in the past few years to address this problem but computation time has been identified as the constraint making the process inefficient. Utilities today are in need of tools, techniques and also the methods that will enable them to predict the dynamic stability and reliability of the grid in the real-time. A power system is secure against a given contingency if it operates within tolerable operating limits before and after the occurrence of the contingency. In practice, it is not possible to secure a power system against all possible contingencies. Therefore, only the most critical contingencies (imminent disturbances) are considered. Contingency ranking attempts to estimate the impact of various contingencies without actually solving the power network. Existing methods of contingency ranking methods suffer from masking effects in approximate methods and slow execution in more accurate ranking methods. This paper presents an exact and precise method of contingency ranking. The method used here takes due consideration of both apparent power overloading and voltage violations simultaneously to find indices which in turn used to rank the contingencies. Here 1P1Q solution is used to find the post contingency voltages and power flows. The proposed work is simulated on IEEE-14 and IEEE- 30 bus test systems in MATLAB environment. The method used is based on realistic approach taking practical situations into account. Besides taking real situations into consideration, this method is fast enough to be considered for on-line security analysis.
\end{abstract}

Keywords: Power system operation, power system security, contingency analysis, power system stability.

\section{INTRODUCTION}

Both the historical and present day civilization of mankind are closely related to energy, and there is no reason to doubt but that in the future our existence will be more and more dependent upon the energy. Electrical energy occupies the top most position in the energy hierarchy. It finds innumerable uses in homes, industry, agriculture and transport.

Besides its use for domestic, industrial and commercial and industrial purposes it is used for defence and agricultural production. Electrical power system is a technical wonder. Electricity and its accessibility are the greatest engineering achievements of the $20^{\text {th }}$ century. Power system is made of interconnected components, each designed to play a critical role for smooth operation of the system at all the times.

It is well known that a power system is a complex network consisting of numerous equipments such as generators, transformers, circuit breakers, transmission lines etc. failure of any of these components during its operation will harm the reliability of the system and hence leading to an outages.

Thus one of the agenda of the power system planning and its operation is to study the effects of outages in terms of its severity. Installation of redundant generation capacity or the transmission lines is essential in order to make the system run even when any of its components fails. But power system being dynamic doesn't guarantee that it will be $100 \%$ reliable. The following reasons make the management of power systems more difficult than earlier.
- Due to increased competition, existing power systems are required to provide greater profit or produce the same service at the lower costs, thereby increasing the duration of power systems operating close to security and stability limits.

- Environmental constraints severely limit the expansion of a transmission network.

- Fewer operators are engaged in the supervision and operation of power system.

- The transmission capacity for all transactions in the open excess network needs to be determined.

Although power generation, transmission and distribution are unbundled, there still exists common interest for these companies: power system adequacy and power system security. The adequacy of production and transmission capacity is maintained in the long-term and is related to power system planning.

The concept of adequacy is generally considered to be the existence of sufficient facilities within the system to satisfy the consumer demand. These facilities include those necessary to generate sufficient energy and associated transmission and distribution networks required to transfer the energy to the actual consumer load points. Adequacy is therefore considered to be associated with the static conditions which do not include the system disturbances.

Security on the other hand, is considered to relate to the ability of the system to respond to the disturbances arising within that system. Security is therefore associated with 
the response of system to whatever disturbances they are subjected. These are considered to be the conditions causing local and widespread effects and the loss of major generation and transmission facilities.

To achieve high degree of reliability and economy, problem of planning and coordinated operation of a vast and complex network have to be solved. This is the main intension of power system studies. For planning the operation, improvement and expansion of power system, a power system engineer needs the load flow studies. An important part of security study therefore, moves around the power systems ability to withstand the effect of contingencies, a contingency can consist of several actions:

- Simple line outage of a single transmission line.

- Complex line outage of several lines, a number of generators.

These problems may lead to total imbalance in the power system, which will bring down the power system to halt state. Hence preventive measure is essential to avoid such situations on the power system.

Contingency analysis being very important and sensitive part of power system security, demands maximum possible accuracy. The number of contingencies in a large power system can be in more than thousands; however the time slot available for power system operator to analyze so many contingencies and take appropriate action to avoid any post contingency violation is quite limited. The constraint of time boundation necessitates screening and ranking of only potential contingencies followed by detailed analysis and proper control actions for credible contingencies. All constrains including flow gate limits need to be respected following any credible contingency.

Contingency analysis, ranking and selection are acceptably considered as crucial activities in power security assessment and normally conducted in line with the voltage stability analysis. Most of contingency analysis algorithms are meant to perform the contingency selection in order to identify and filter out worst contingency cases for further detailed analysis once the preventive and corrective measures have been identified. Complex system mainly caused by the economic and environmental pressures in continuing interconnections of bulk power systems has caused the system to operate close to its limit of stability. This situation becomes worst when contingencies occur in the stressed power network. Contingencies caused by line, generator and transformer outages are identified as the most common contingencies that could violate the voltage stability condition of the entire system.

\section{METHODOLOGY}

\section{A. Exact ranking technique}

This method aims at finding the exact number of possible violations following a contingency in power system. The logic behind this is to have contribution of ' 1 ' by violated line/bus and ' 0 ' by non-violated line/bus to ranking index named as exact ranking index (ERI) as given in eqn.

$$
E_{R I}=\sum_{l} \text { All branches } R I_{\mathrm{S}}+\sum_{i} \text { All branches } R I_{\mathrm{v}}
$$

Where $R I_{s}$ : Ranking index of apparent power flow $S_{l}$ of line

$$
R I_{\mathrm{s}}=\left\{\begin{array}{l}
1 ; \text { for } S_{l}>P_{l}^{\max } \\
0 ; \text { for } S_{l}<P_{l}^{\max }
\end{array}\right.
$$

Ranking index of voltage bus

$$
R I_{\mathrm{v}}:
$$

$$
R I_{\mathrm{v}}=\left\{\begin{array}{l}
1 ; \text { for }\left|E_{\text {nom }}-E_{i}\right|>\Delta E_{i}^{\text {max }} \\
0 ; \text { for }\left|E_{\text {nom }}-E_{i}\right|<\Delta E_{i}^{\text {max }}
\end{array}\right.
$$

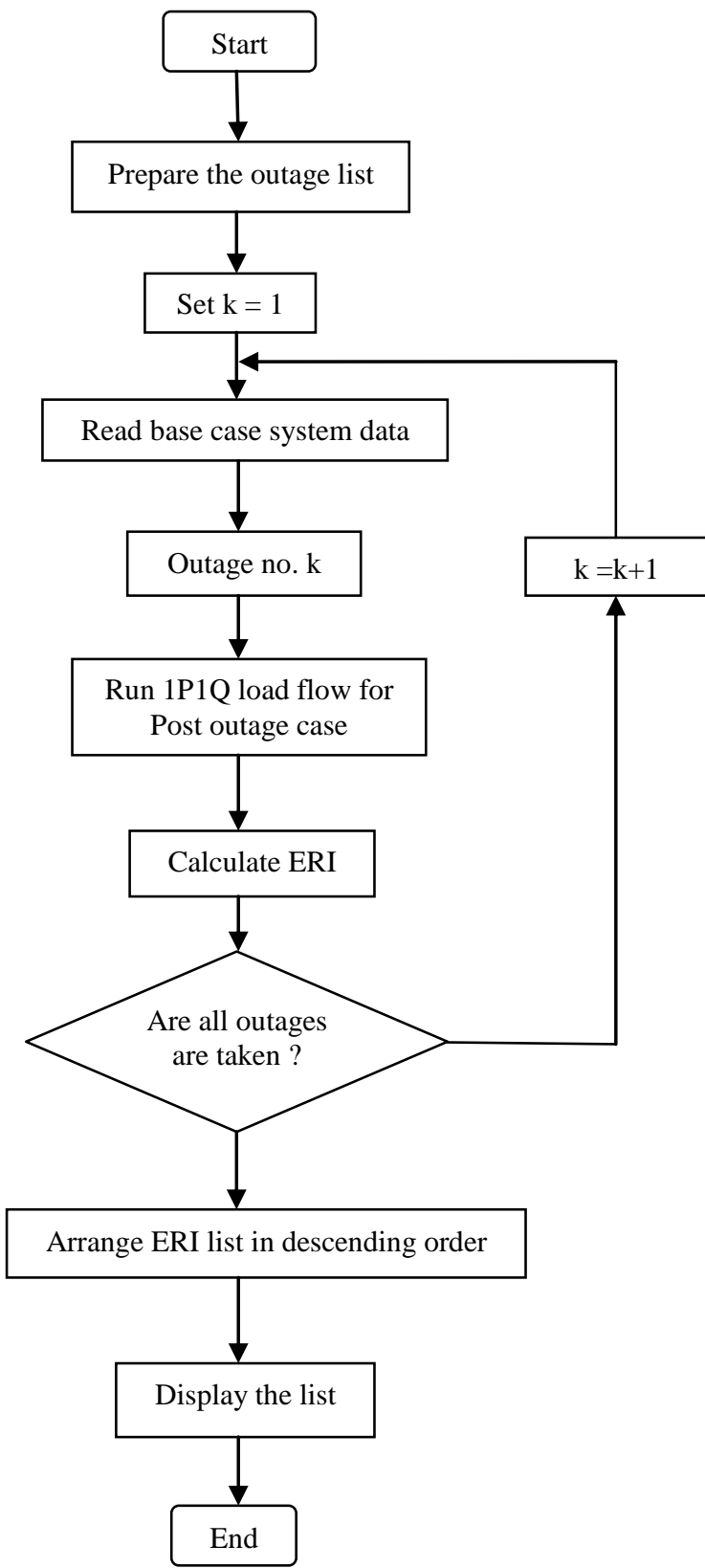

Fig 1: Flow chart for exact contingency ranking 
B. Precise Ranking Technique

Exact ranking method has presented fairly acceptable and correct results, however it doesn't differentiate between the outages having same severity and hence label them with same ranking. This technique hereafter called precise ranking technique addresses the concern of identical ranking for outages having the same severity and takes into account in case there is any line or bus reaching near to its limit following a particular contingency. This ranking is based on new index hereafter called as precise ranking index (PRI) given in eqn.

$$
P_{R I}=\sum_{\text {All branches }} R I_{\mathrm{s}}+\sum_{\text {All branches }} R I_{\mathrm{v}}
$$

Where $R I_{S}$ : Ranking index of apparent power flow

$S_{l}$ of the line

$$
R I_{\mathrm{s}}=\left\{\begin{array}{l}
1 ; \quad \text { for } S_{l}>P_{l}^{\max } \\
\left(\frac{P_{\text {flowl }}}{P_{l}^{\text {max }}}\right)^{2 n} ; \text { for } S_{l}<P_{l}^{\max }
\end{array}\right.
$$

$R I_{\mathrm{v}}:$ Ranking index of voltage bus

$$
R I_{\mathrm{v}}= \begin{cases}1 & ; \text { for }\left|E_{\text {nom }}-E_{i}\right|>\Delta E_{i}^{\max } \\ \left(\frac{\left|E_{n o m}-E_{i}\right|}{\Delta E^{\max }}\right)^{2 m} & ; \text { for }\left|E_{\text {nom }}-E_{i}\right|<\Delta E_{i}^{\max }\end{cases}
$$

Where $E_{\text {nom }}$ is nominal voltage of bus

$P_{\text {flowl }} \quad$ is real power flow in line $l$.

$P_{l}^{\max }$ maximum loading capacity of line $l$.

$\Delta E_{i} \quad$ is the difference between voltage magnitude after 1P1Q solution an base case voltage magnitude at bus i.

$\Delta E^{\max } \quad$ is a value set by utility experts indicating how much maximum voltage deviation is allowed at any bus.

$m, n \quad$ are integers

As in the precise ranking method we are considering the actual value of power flow in the transmission line and actual value of bus voltages simultaneously for finding the index values to rank the contingencies according to severity. This precise ranking method clearly ranks all the contingencies according to severity. The procedure for this technique remains same

\section{Algorithm for precise ranking indices}

Step 1: Enter the system data; define R, X, sending end bus and receiving end bus number, line data, bus data, tolerance limit etc.

Step 2: Prepare the outage list. The outage list consists of all the line outages.

Step 3: Now read the base case system data.
Step 4: Now pick an outage say $\mathrm{k}$ from the outage list and remove that outage from outage list.

Step 5: Now run 1P1Q load flow for the post outage case.

Step 6: Calculate the precise ranking indices.

Step 7: Repeat the process from step 2 to step 6 until all outages are considered.

Step 8: Now arrange the precise ranking indices in the descending order

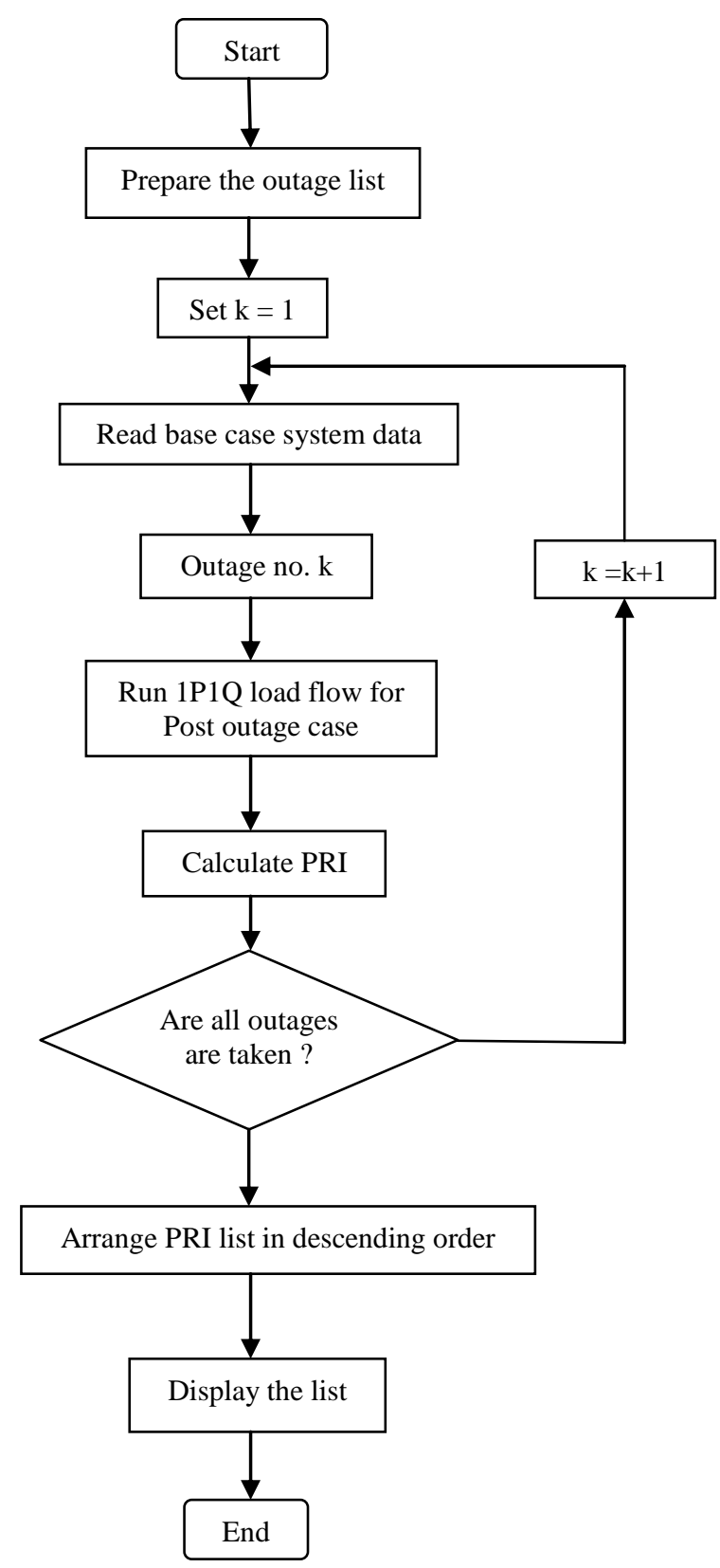

Fig 2: Flow chart for precise contingency ranking 


\section{RESULTS AND DISCUSSION}

MATLAB software has been utilized to write the programs to identify the contingency which is most severe. Here exact ranking method and precise ranking method is used to find the exact ranking indices (ERI) and precise ranking indices (PRI) respectively.

The exact ranking indices (ERI) and precise ranking indices (PRI) are calculated for all the line outages separately. Then these indices are used to rank each contingency. The indices with the highest value indicate that particular contingency as most severe and the indices with the lowest value indicate that contingency as less severe.

This method is applied on the IEEE 14 bus test system and the results are as follows.

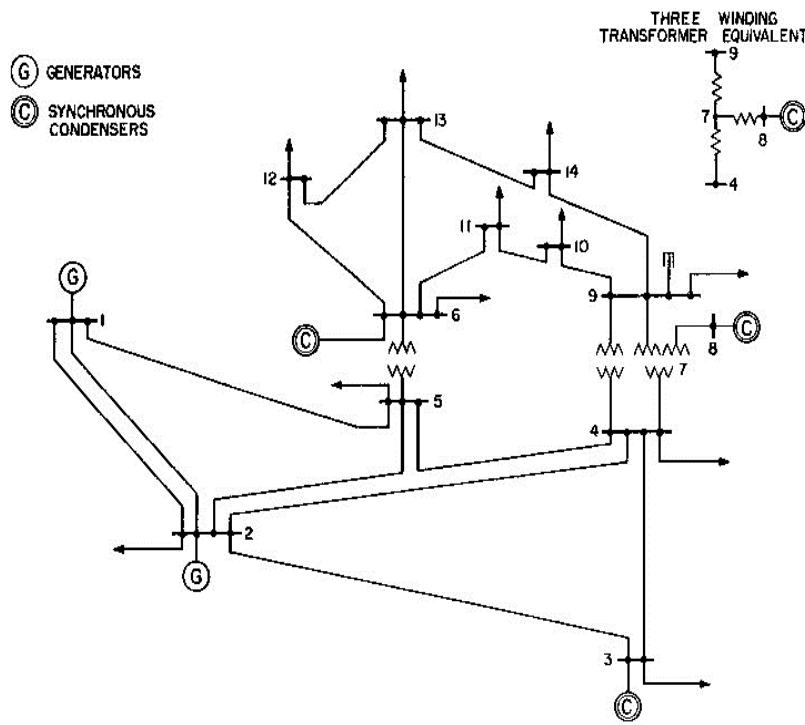

Fig 3: IEEE - 14 bus test system

TABLE I

ERI BASED CONTINGENCY RANKING

\begin{tabular}{|c|c|c|c|}
\hline S. No & Outage & ERI & $\begin{array}{c}\text { ERI } \\
\text { based } \\
\text { ranking }\end{array}$ \\
\hline 1 & $\begin{array}{c}\text { Line 1 } \\
\text { (bus1-bus2) }\end{array}$ & 1 & 8 \\
\hline 2 & $\begin{array}{c}\text { Line 2 } \\
\text { (bus1-bus5) }\end{array}$ & 7 & 2 \\
\hline 3 & $\begin{array}{c}\text { Line 3 } \\
\text { (bus2-bus3) }\end{array}$ & 6 & 3 \\
\hline 5 & $\begin{array}{c}\text { Line 4 } \\
\text { (bus2-bus4) }\end{array}$ & 7 & 2 \\
\hline 6 & $\begin{array}{c}\text { Line 5 } \\
\text { (bus2-bus5) }\end{array}$ & 7 & 8 \\
\hline 7 & $\begin{array}{c}\text { Line 6 } \\
\text { Lus3-bus4) } 7\end{array}$ & 2 & 7 \\
\hline 8 & $\begin{array}{c}\text { Line } 8 \\
\text { (bus4-bus7) }\end{array}$ & 9 & 1 \\
\hline 9 & $\begin{array}{c}\text { Line 9 } \\
\text { (bus4-bus9) }\end{array}$ & 6 & 3 \\
\hline
\end{tabular}

\begin{tabular}{|c|c|c|c|}
\hline 10 & $\begin{array}{c}\text { Line } 10 \\
\text { (bus5-bus6) }\end{array}$ & 9 & 1 \\
\hline 11 & $\begin{array}{c}\text { Line } 11 \\
\text { (bus6-bus11) }\end{array}$ & 3 & 6 \\
\hline 12 & $\begin{array}{c}\text { Line } 12 \\
\text { (bus6-bus12) }\end{array}$ & 3 & 6 \\
\hline 13 & $\begin{array}{c}\text { Line } 13 \\
\text { (bus6-bus13) }\end{array}$ & 4 & 5 \\
\hline 14 & $\begin{array}{c}\text { Line } 14 \\
\text { (bus7-bus8) }\end{array}$ & 1 & 8 \\
\hline 15 & $\begin{array}{c}\text { Line } 15 \\
\text { (bus7-bus9) }\end{array}$ & 7 & 2 \\
\hline 16 & $\begin{array}{c}\text { Line } 16 \\
\text { (bus9-bus10) }\end{array}$ & 5 & 4 \\
\hline 17 & $\begin{array}{c}\text { Line } 17 \\
\text { (bus9-bus14) }\end{array}$ & 3 & 6 \\
\hline 18 & $\begin{array}{l}\text { Line } 18 \\
\text { (bus10- } \\
\text { bus11) }\end{array}$ & 2 & 7 \\
\hline 19 & $\begin{array}{l}\text { Line } 19 \\
\text { (bus12- } \\
\text { bus13) }\end{array}$ & 2 & 7 \\
\hline 20 & $\begin{array}{l}\text { Line } 20 \\
\text { (bus13- } \\
\text { bus14) }\end{array}$ & 1 & 8 \\
\hline
\end{tabular}

TABLE II

PRI BASED CONTINGENCY RANKING

\begin{tabular}{|c|c|c|c|}
\hline S. No & Outage & PRI & $\begin{array}{c}\text { PRI } \\
\text { based } \\
\text { ranking }\end{array}$ \\
\hline 1 & $\begin{array}{c}\text { Line 1 } \\
\text { (bus1-bus2) }\end{array}$ & 25.0948 & 17 \\
\hline 2 & $\begin{array}{c}\text { Line } 2 \\
\text { (bus1-bus5) } \\
\end{array}$ & 27.6835 & 4 \\
\hline 3 & $\begin{array}{c}\text { Line } 3 \\
\text { (bus2-bus3) }\end{array}$ & 27.2959 & 8 \\
\hline 4 & $\begin{array}{c}\text { Line } 4 \\
\text { (bus2-bus4) }\end{array}$ & 27.9477 & 3 \\
\hline 5 & $\begin{array}{c}\text { Line } 5 \\
\text { (bus2-bus5) }\end{array}$ & 27.5531 & 5 \\
\hline 6 & $\begin{array}{c}\text { Line } 6 \\
\text { (bus3-bus4) }\end{array}$ & 24.3333 & 19 \\
\hline 7 & $\begin{array}{c}\text { Line } 7 \\
\text { (bus4-bus5) } \\
\end{array}$ & 25.5544 & 15 \\
\hline 8 & $\begin{array}{c}\text { Line } 8 \\
\text { (bus4-bus7) }\end{array}$ & 29.0930 & 2 \\
\hline 9 & $\begin{array}{c}\text { Line } 9 \\
\text { (bus4-bus9) }\end{array}$ & 27.3671 & 7 \\
\hline 10 & $\begin{array}{c}\text { Line } 10 \\
\text { (bus5-bus6) }\end{array}$ & 29.0931 & 1 \\
\hline 11 & $\begin{array}{c}\text { Line 11 } \\
\text { (bus6-bus11) }\end{array}$ & 25.2636 & 13 \\
\hline 12 & $\begin{array}{c}\text { Line } 12 \\
\text { (bus6-bus12) }\end{array}$ & 25.5397 & 11 \\
\hline 13 & $\begin{array}{c}\text { Line 13 } \\
\text { (bus6-bus13) }\end{array}$ & 25.7627 & 10 \\
\hline 14 & $\begin{array}{c}\text { Line } 14 \\
\text { (bus7-bus8) } \\
\end{array}$ & 25.0948 & 17 \\
\hline
\end{tabular}



INTERNATIONAL JOURNAL OF INNOVATIVE RESEARCH IN ELECTRICAL, ELECTRONICS, INSTRUMENTATION AND CONTROL ENGINEERING Vol. 3, Issue 5, May 2015

\begin{tabular}{|c|c|c|c|}
\hline 15 & $\begin{array}{c}\text { Line 15 } \\
\text { (bus7-bus9) }\end{array}$ & 27.4930 & 6 \\
\hline 16 & $\begin{array}{c}\text { Line 16 } \\
\text { (bus9-bus10) }\end{array}$ & 26.0827 & 9 \\
\hline 17 & $\begin{array}{c}\text { Line 17 } \\
\text { (bus9-bus14) }\end{array}$ & 25.3655 & 12 \\
\hline 18 & $\begin{array}{c}\text { Line 18 } \\
\text { (bus10- } \\
\text { bus11) }\end{array}$ & 24.9831 & 14 \\
\hline 19 & $\begin{array}{c}\text { Line 19 } \\
\text { (bus12- } \\
\text { bus13) }\end{array}$ & 25.0195 & 16 \\
\hline 20 & $\begin{array}{c}\text { Line 20 } \\
\text { (bus13- } \\
\text { bus14) }\end{array}$ & 24.7289 & 18 \\
\hline
\end{tabular}

\begin{tabular}{|c|c|c|c|}
\hline 18 & $\begin{array}{c}\text { Line 14 } \\
\text { (bus7-bus8) }\end{array}$ & 8 & 17 \\
\hline 19 & $\begin{array}{c}\text { Line 20 } \\
\text { (bus13- } \\
\text { bus14) }\end{array}$ & 8 & 18 \\
\hline 20 & $\begin{array}{c}\text { Line 6 } \\
\text { (bus3-bus4) }\end{array}$ & 8 & 19 \\
\hline
\end{tabular}

TABLE III

COMPARISON OF ERI BASED CONTINGENCY RANKING AND PRI BASED CONTINGENCY RANKING

\begin{tabular}{|c|c|c|c|}
\hline S. No & Outage & $\begin{array}{c}\text { ERI } \\
\text { based } \\
\text { ranking }\end{array}$ & $\begin{array}{c}\text { PRI } \\
\text { based } \\
\text { ranking }\end{array}$ \\
\hline 1 & $\begin{array}{c}\text { Line } 10 \\
\text { (bus5-bus6) }\end{array}$ & 1 & 1 \\
\hline 2 & $\begin{array}{c}\text { Line } 8 \\
\text { (bus4-bus7) }\end{array}$ & 1 & 2 \\
\hline 3 & $\begin{array}{c}\text { Line } 4 \\
\text { (bus2-bus4) }\end{array}$ & 2 & 3 \\
\hline 4 & $\begin{array}{c}\text { Line } 2 \\
\text { (bus1-bus5) }\end{array}$ & 2 & 4 \\
\hline 5 & $\begin{array}{c}\text { Line } 5 \\
\text { (bus2-bus5) }\end{array}$ & 2 & 5 \\
\hline 6 & $\begin{array}{c}\text { Line } 15 \\
\text { (bus7-bus9) }\end{array}$ & 2 & 6 \\
\hline 7 & $\begin{array}{c}\text { Line } 9 \\
\text { (bus4-bus9) }\end{array}$ & 3 & 7 \\
\hline 8 & $\begin{array}{c}\text { Line } 3 \\
\text { (bus2-bus3) }\end{array}$ & 3 & 8 \\
\hline 9 & $\begin{array}{c}\text { Line 16 } \\
\text { (bus9-bus10) }\end{array}$ & 4 & 9 \\
\hline 10 & $\begin{array}{c}\text { Line 13 } \\
\text { (bus6-bus13) }\end{array}$ & 5 & 10 \\
\hline 11 & $\begin{array}{c}\text { Line 12 } \\
\text { (bus6-bus12) }\end{array}$ & 6 & 11 \\
\hline 12 & $\begin{array}{c}\text { Line } 17 \\
\text { (bus9-bus14) }\end{array}$ & 6 & 12 \\
\hline 13 & $\begin{array}{c}\text { Line 11 } \\
\text { (bus6-bus11) }\end{array}$ & 6 & 13 \\
\hline 14 & $\begin{array}{l}\text { Line 18 } \\
\text { (bus10- } \\
\text { bus11) }\end{array}$ & 7 & 14 \\
\hline 15 & $\begin{array}{c}\text { Line } 7 \\
\text { (bus4-bus5) } \\
\end{array}$ & 7 & 15 \\
\hline 16 & $\begin{array}{l}\text { Line 19 } \\
\text { (bus12- } \\
\text { bus13) }\end{array}$ & 7 & 16 \\
\hline 17 & $\begin{array}{c}\text { Line } 1 \\
\text { (bus1-bus2) }\end{array}$ & 8 & 17 \\
\hline
\end{tabular}

The results for IEEE - 14 bus test system are obtained. The ranking indices are obtained for all the outages. The index which is having highest value indicates the contingency as most severe and the index with lowest value indicates the contingency as less severe.

It is seen from the results of exact ranking method that, the line 8 and line 10 outage both are having same value of ERI as 9. It implies that these are the contingencies with more severity and whose outages severely affect the operation of power system hence these contingencies are ranked as 1 . The line 1 , line 6 , line 14 , line 20 outages are having same value of ERI as 1 . It implies that these are the contingencies with less severity.

Similarly from the precise ranking method it is seen that, the line 10 outage has the PRI as 29.0931 and for the line 8 has the PRI as 29.0930. Hence the line outage 10 is ranked as 1 and line 8 as 2 . This indicates that in the exact ranking indices, where the severity of line 10 and line 8 outages is same, the precise ranking indices distinguish between the two.

Finally the comparison of exact ranking indices (ERI) and precise ranking indices (PRI) based contingency ranking is made in the table. It is seen from the table that, the exact ranking method does not differentiate between the outages with same severity hence label them with identical ranking. The precise ranking technique addresses the concern of identical ranking for the outages with same severity by taking into account in case there is any line or bus reaching near to its limits following a particular contingency. Hence precise ranking method distinguishes between the outages having same severity.

This method is applied on the IEEE 30 bus test system and the results are as follows.

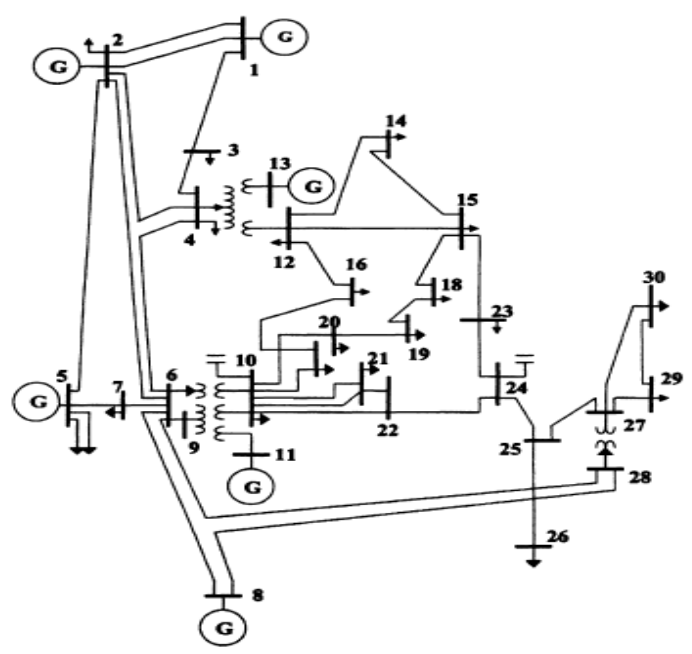

Fig 4: IEEE - 30 bus test system 
TABLE IV

ERI BASED CONTINGENCY RANKING

\begin{tabular}{|c|c|c|c|}
\hline S. No & Outage & ERI & $\begin{array}{c}\text { ERI } \\
\text { based } \\
\text { ranking }\end{array}$ \\
\hline 1 & $\begin{array}{c}\text { Line } 1 \\
\text { (bus1-bus2) }\end{array}$ & 7 & 10 \\
\hline 2 & $\begin{array}{c}\text { Line } 2 \\
\text { (bus1-bus3) }\end{array}$ & 7 & 10 \\
\hline 3 & $\begin{array}{c}\text { Line } 3 \\
\text { (bus2-bus4) }\end{array}$ & 7 & 10 \\
\hline 4 & $\begin{array}{c}\text { Line } 4 \\
\text { (bus2-bus5) }\end{array}$ & 7 & 10 \\
\hline 5 & $\begin{array}{c}\text { Line } 5 \\
\text { (bus2-bus6) }\end{array}$ & 6 & 11 \\
\hline 6 & $\begin{array}{c}\text { Line } 6 \\
\text { (bus3-bus4) }\end{array}$ & 7 & 10 \\
\hline 7 & $\begin{array}{c}\text { Line } 7 \\
\text { (bus4-bus6) }\end{array}$ & 20 & 4 \\
\hline 8 & $\begin{array}{c}\text { Line } 8 \\
\text { (bus4-bus12) }\end{array}$ & 25 & 2 \\
\hline 9 & $\begin{array}{c}\text { Line } 9 \\
\text { (bus5-bus7) }\end{array}$ & 6 & 11 \\
\hline 10 & $\begin{array}{c}\text { Line } 10 \\
\text { (bus6-bus7) }\end{array}$ & 7 & 10 \\
\hline 11 & $\begin{array}{c}\text { Line } 11 \\
\text { (bus6-bus8) }\end{array}$ & 7 & 10 \\
\hline 12 & $\begin{array}{c}\text { Line } 12 \\
\text { (bus6-bus9) }\end{array}$ & 21 & 3 \\
\hline 13 & $\begin{array}{c}\text { Line } 13 \\
\text { (bus6-bus10) }\end{array}$ & 12 & 6 \\
\hline 14 & $\begin{array}{c}\text { Line } 14 \\
\text { (bus6-bus28) }\end{array}$ & 7 & 10 \\
\hline 15 & $\begin{array}{c}\text { Line } 15 \\
\text { (bus8-bus28) }\end{array}$ & 7 & 10 \\
\hline 16 & $\begin{array}{c}\text { Line } 16 \\
\text { (bus9-bus11) }\end{array}$ & 9 & 8 \\
\hline 17 & $\begin{array}{c}\text { Line } 17 \\
\text { (bus9-bus10) }\end{array}$ & 7 & 10 \\
\hline 18 & $\begin{array}{c}\text { Line } 18 \\
\text { (bus10-bus20) }\end{array}$ & 10 & 7 \\
\hline 19 & $\begin{array}{c}\text { Line } 19 \\
\text { (bus10-bus17) }\end{array}$ & 7 & 10 \\
\hline 20 & $\begin{array}{c}\text { Line } 20 \\
\text { (bus10-bus21) }\end{array}$ & 7 & 10 \\
\hline 21 & $\begin{array}{c}\text { Line } 21 \\
\text { (bus10-bus22) }\end{array}$ & 7 & 10 \\
\hline 22 & $\begin{array}{c}\text { Line } 22 \\
\text { (bus12-bus13) }\end{array}$ & 31 & 1 \\
\hline 23 & $\begin{array}{c}\text { Line } 23 \\
\text { (bus12-bus14) }\end{array}$ & 7 & 10 \\
\hline 24 & $\begin{array}{c}\text { Line } 24 \\
\text { (bus12-bus15) }\end{array}$ & 12 & 6 \\
\hline 25 & $\begin{array}{c}\text { Line } 25 \\
\text { (bus12-bus16) }\end{array}$ & 7 & 10 \\
\hline 26 & $\begin{array}{c}\text { Line } 26 \\
\text { (bus14-bus15) }\end{array}$ & 7 & 10 \\
\hline 27 & $\begin{array}{c}\text { Line } 27 \\
\text { (bus15-bus18) }\end{array}$ & 10 & 7 \\
\hline
\end{tabular}

\begin{tabular}{|c|c|c|c|}
\hline 28 & $\begin{array}{c}\text { Line } 28 \\
\text { (bus15-bus23) }\end{array}$ & 8 & 9 \\
\hline 29 & $\begin{array}{c}\text { Line } 29 \\
\text { (bus16-bus17) }\end{array}$ & 7 & 10 \\
\hline 30 & $\begin{array}{c}\text { Line } 30 \\
\text { (bus18-bus19) }\end{array}$ & 8 & 9 \\
\hline 31 & $\begin{array}{c}\text { Line } 31 \\
\text { (bus19-bus20) }\end{array}$ & 9 & 8 \\
\hline 32 & $\begin{array}{c}\text { Line } 32 \\
\text { (bus21-bus22) }\end{array}$ & 7 & 10 \\
\hline 33 & $\begin{array}{c}\text { Line } 33 \\
\text { (bus22-bus24) }\end{array}$ & 7 & 10 \\
\hline 34 & $\begin{array}{c}\text { Line } 34 \\
\text { (bus23-bus24) }\end{array}$ & 8 & 9 \\
\hline 35 & $\begin{array}{c}\text { Line } 35 \\
\text { (bus24-bus25) }\end{array}$ & 7 & 10 \\
\hline 36 & $\begin{array}{c}\text { Line } 36 \\
\text { (bus25-bus26) }\end{array}$ & 7 & 10 \\
\hline 37 & $\begin{array}{c}\text { Line } 37 \\
\text { (bus25-bus27) }\end{array}$ & 10 & 7 \\
\hline 38 & $\begin{array}{c}\text { Line } 38 \\
\text { (bus27-bus29) }\end{array}$ & 7 & 10 \\
\hline 39 & $\begin{array}{c}\text { Line } 39 \\
\text { (bus27-bus30) }\end{array}$ & 7 & 10 \\
\hline 40 & $\begin{array}{c}\text { Line } 40 \\
\text { (bus28-bus27) }\end{array}$ & 17 & 5 \\
\hline 41 & $\begin{array}{c}\text { Line } 41 \\
\text { (bus29-bus30) }\end{array}$ & 7 & 10 \\
\hline
\end{tabular}

TABLE V

PRI BASED CONTINGENCY RANKING

\begin{tabular}{|c|c|c|c|}
\hline S. No & Outage & PRI & $\begin{array}{c}\text { PRI } \\
\text { based } \\
\text { ranking }\end{array}$ \\
\hline 1 & $\begin{array}{c}\text { Line } 1 \\
\text { (bus1-bus2) }\end{array}$ & 14.0577 & 35 \\
\hline 2 & $\begin{array}{c}\text { Line } 2 \\
\text { (bus1-bus3) }\end{array}$ & 14.3998 & 34 \\
\hline 3 & $\begin{array}{c}\text { Line } 3 \\
\text { (bus2-bus4) }\end{array}$ & 14.4636 & 33 \\
\hline 4 & $\begin{array}{c}\text { Line } 4 \\
\text { (bus2-bus5) }\end{array}$ & 15.7486 & 19 \\
\hline 5 & $\begin{array}{c}\text { Line } 5 \\
\text { (bus2-bus6) }\end{array}$ & 13.0393 & 38 \\
\hline 6 & $\begin{array}{c}\text { Line } 6 \\
\text { (bus3-bus4) }\end{array}$ & 14.9873 & 30 \\
\hline 7 & $\begin{array}{c}\text { Line } 7 \\
\text { (bus4-bus6) }\end{array}$ & 27.8616 & 4 \\
\hline 8 & $\begin{array}{l}\text { Line } 8 \\
\text { (bus4- } \\
\text { bus12) }\end{array}$ & 30.1612 & 2 \\
\hline 9 & $\begin{array}{c}\text { Line } 9 \\
\text { (bus5-bus7) }\end{array}$ & 13.5283 & 37 \\
\hline 10 & $\begin{array}{c}\text { Line } 10 \\
\text { (bus6-bus7) }\end{array}$ & 15.2398 & 27 \\
\hline 11 & $\begin{array}{c}\text { Line } 11 \\
\text { (bus6-bus8) }\end{array}$ & 15.7466 & 20 \\
\hline 12 & $\begin{array}{c}\text { Line } 12 \\
\text { (bus6-bus9) }\end{array}$ & 28.1640 & 3 \\
\hline
\end{tabular}


INTERNATIONAL JOURNAL OF INNOVATIVE RESEARCH IN ELECTRICAL, ELECTRONICS, INSTRUMENTATION AND CONTROL ENGINEERING Vol. 3, Issue 5, May 2015

\begin{tabular}{|c|c|c|c|c|c|c|c|}
\hline 13 & $\begin{array}{c}\text { Line } 13 \\
\text { (bus6- } \\
\text { bus10) }\end{array}$ & 20.9154 & 7 & 33 & $\begin{array}{l}\text { Line } 33 \\
\text { (bus22- } \\
\text { bus24) }\end{array}$ & 15.7198 & 21 \\
\hline 14 & $\begin{array}{c}\text { Line } 14 \\
\text { (bus6- } \\
\text { bus28) }\end{array}$ & 15.0411 & 29 & 34 & $\begin{array}{l}\text { Line } 34 \\
\text { (bus23- } \\
\text { bus24) }\end{array}$ & 16.7296 & 14 \\
\hline 15 & $\begin{array}{c}\text { Line } 15 \\
\text { (bus8- } \\
\text { bus28) }\end{array}$ & 14.9300 & 31 & 35 & $\begin{array}{l}\text { Line } 35 \\
\text { (bus24- } \\
\text { bus25) }\end{array}$ & 14.0501 & 36 \\
\hline 16 & $\begin{array}{c}\text { Line } 16 \\
\text { (bus9- } \\
\text { bus11) }\end{array}$ & 18.6769 & 11 & 36 & $\begin{array}{c}\text { Line } 36 \\
\text { (bus25- } \\
\text { bus26) }\end{array}$ & 15.7701 & 18 \\
\hline 17 & $\begin{array}{c}\text { Line } 17 \\
\text { (bus9- } \\
\text { bus10) }\end{array}$ & 15.4983 & 25 & 37 & $\begin{array}{l}\text { Line } 37 \\
\text { (bus } 25- \\
\text { bus } 27 \text { ) }\end{array}$ & 20.2553 & 9 \\
\hline 18 & $\begin{array}{l}\text { Line } 18 \\
\text { (bus10- } \\
\text { bus20) }\end{array}$ & 20.2036 & 10 & 38 & $\begin{array}{l}\text { Line } 38 \\
\text { (bus27- } \\
\text { bus29) }\end{array}$ & 15.9111 & 16 \\
\hline 19 & $\begin{array}{l}\text { Line } 19 \\
\text { (bus10- } \\
\text { bus } 17 \text { ) }\end{array}$ & 14.4636 & 33 & 39 & $\begin{array}{l}\text { Line } 39 \\
\text { (bus27- } \\
\text { bus30) }\end{array}$ & 15.4611 & 26 \\
\hline 20 & $\begin{array}{l}\text { Line } 20 \\
\text { (bus } 10- \\
\text { bus } 21 \text { ) }\end{array}$ & 15.1057 & 28 & 40 & $\begin{array}{l}\text { Line } 40 \\
\text { (bus28- } \\
\text { bus } 27 \text { ) }\end{array}$ & 26.5737 & 5 \\
\hline 21 & $\begin{array}{l}\text { Line } 21 \\
\text { (bus10- } \\
\text { bus22) }\end{array}$ & 15.6828 & 22 & 41 & $\begin{array}{l}\text { Line } 41 \\
\text { (bus29- } \\
\text { bus30) }\end{array}$ & 14.8963 & 32 \\
\hline
\end{tabular}

\begin{tabular}{|c|c|c|c|}
\hline 22 & $\begin{array}{c}\text { Line } 22 \\
\text { (bus12- } \\
\text { bus13) }\end{array}$ & 120.9750 & 1 \\
\hline 23 & $\begin{array}{l}\text { Line } 23 \\
\text { (bus12- } \\
\text { bus14) }\end{array}$ & 15.8882 & 17 \\
\hline 24 & $\begin{array}{l}\text { Line } 24 \\
\text { (bus12- } \\
\text { bus15) }\end{array}$ & 20.9496 & 6 \\
\hline 25 & $\begin{array}{l}\text { Line } 25 \\
\text { (bus } 12- \\
\text { bus16) }\end{array}$ & 15.5513 & 23 \\
\hline 26 & $\begin{array}{c}\text { Line } 26 \\
\text { (bus14- } \\
\text { bus15) }\end{array}$ & 14.4636 & 33 \\
\hline 27 & $\begin{array}{l}\text { Line } 27 \\
\text { (bus15- } \\
\text { bus18) }\end{array}$ & 20.6460 & 8 \\
\hline 28 & $\begin{array}{l}\text { Line } 28 \\
\text { (bus15- } \\
\text { bus23) }\end{array}$ & 17.7011 & 15 \\
\hline 29 & $\begin{array}{c}\text { Line } 29 \\
\text { (bus16- } \\
\text { bus } 17 \text { ) }\end{array}$ & 15.5368 & 24 \\
\hline 30 & $\begin{array}{l}\text { Line } 30 \\
\text { (bus18- } \\
\text { bus19) }\end{array}$ & 16.7882 & 13 \\
\hline 31 & $\begin{array}{l}\text { Line } 31 \\
\text { (bus19- } \\
\text { bus20) }\end{array}$ & 18.3579 & 12 \\
\hline 32 & $\begin{array}{l}\text { Line } 32 \\
\text { (bus21- } \\
\text { bus22) }\end{array}$ & 15.5513 & 23 \\
\hline
\end{tabular}

TABLE VI

COMPARISON OF ERI BASED CONTINGENCY RANKING AND PRI BASED CONTINGENCY RANKING

\begin{tabular}{|c|c|c|c|}
\hline S. No & Outage & $\begin{array}{c}\text { ERI } \\
\text { based } \\
\text { ranking }\end{array}$ & $\begin{array}{c}\text { PRI } \\
\text { based } \\
\text { ranking }\end{array}$ \\
\hline 1 & $\begin{array}{c}\text { Line } 22 \\
\text { (bus12- } \\
\text { bus 13) }\end{array}$ & 1 & 1 \\
\hline 2 & $\begin{array}{c}\text { Line } 8 \\
\text { (bus4-bus12) }\end{array}$ & 2 & 2 \\
\hline 3 & $\begin{array}{c}\text { Line } 12 \\
\text { (bus6-bus9) }\end{array}$ & 3 & 3 \\
\hline 4 & $\begin{array}{c}\text { Line } 7 \\
\text { (bus4-bus6) }\end{array}$ & 4 & 4 \\
\hline 5 & $\begin{array}{l}\text { Line } 40 \\
\text { (bus28- } \\
\text { bus27) }\end{array}$ & 5 & 5 \\
\hline 6 & $\begin{array}{c}\text { Line } 24 \\
\text { (bus } 12- \\
\text { bus } 15)\end{array}$ & 6 & 6 \\
\hline 7 & $\begin{array}{c}\text { Line 13 } \\
\text { (bus6-bus10) }\end{array}$ & 6 & 7 \\
\hline 8 & $\begin{array}{l}\text { Line } 27 \\
\text { (bus } 15- \\
\text { bus } 18)\end{array}$ & 7 & 8 \\
\hline 9 & $\begin{array}{l}\text { Line } 37 \\
\text { (bus } 25- \\
\text { bus } 27 \text { ) }\end{array}$ & 7 & 9 \\
\hline 10 & $\begin{array}{l}\text { Line } 18 \\
\text { (bus } 10- \\
\text { bus } 20 \text { ) }\end{array}$ & 7 & 10 \\
\hline
\end{tabular}




\begin{tabular}{|c|c|c|c|}
\hline 11 & $\begin{array}{c}\text { Line } 16 \\
\text { (bus9-bus11) }\end{array}$ & 8 & 11 \\
\hline 12 & $\begin{array}{l}\text { Line } 31 \\
\text { (bus19- } \\
\text { bus20) }\end{array}$ & 8 & 12 \\
\hline 13 & $\begin{array}{l}\text { Line } 30 \\
\text { (bus18- } \\
\text { bus19) }\end{array}$ & 9 & 13 \\
\hline 14 & $\begin{array}{l}\text { Line } 34 \\
\text { (bus23- } \\
\text { bus24) }\end{array}$ & 9 & 14 \\
\hline 15 & $\begin{array}{l}\text { Line } 28 \\
\text { (bus15- } \\
\text { bus23) }\end{array}$ & 9 & 15 \\
\hline 16 & $\begin{array}{l}\text { Line } 38 \\
\text { (bus27- } \\
\text { bus29) }\end{array}$ & 10 & 16 \\
\hline 17 & $\begin{array}{l}\text { Line } 23 \\
\text { (bus12- } \\
\text { bus14) }\end{array}$ & 10 & 17 \\
\hline 18 & $\begin{array}{l}\text { Line } 36 \\
\text { (bus25- } \\
\text { bus26) }\end{array}$ & 10 & 18 \\
\hline 19 & $\begin{array}{c}\text { Line } 4 \\
\text { (bus2-bus5) } \\
\end{array}$ & 10 & 19 \\
\hline 20 & $\begin{array}{c}\text { Line } 11 \\
\text { (bus6-bus8) }\end{array}$ & 10 & 20 \\
\hline 21 & $\begin{array}{l}\text { Line } 33 \\
\text { (bus22- } \\
\text { bus24) }\end{array}$ & 10 & 21 \\
\hline 22 & $\begin{array}{l}\text { Line } 21 \\
\text { (bus10- } \\
\text { bus22) }\end{array}$ & 10 & 22 \\
\hline 23 & $\begin{array}{l}\text { Line } 32 \\
\text { (bus21- } \\
\text { bus22) }\end{array}$ & 10 & 23 \\
\hline 24 & $\begin{array}{l}\text { Line } 25 \\
\text { (bus12- } \\
\text { bus16) }\end{array}$ & 10 & 23 \\
\hline 25 & $\begin{array}{c}\text { Line } 29 \\
\text { (bus16- } \\
\text { bus17) }\end{array}$ & 10 & 24 \\
\hline 26 & $\begin{array}{c}\text { 'Line } 17 \\
\text { (bus9-bus10) }\end{array}$ & 10 & 25 \\
\hline 27 & $\begin{array}{l}\text { Line } 39 \\
\text { (bus27- } \\
\text { bus30) }\end{array}$ & 10 & 26 \\
\hline 28 & $\begin{array}{c}\text { Line } 10 \\
\text { (bus6-bus7) }\end{array}$ & 10 & 27 \\
\hline 29 & $\begin{array}{l}\text { Line } 20 \\
\text { (bus10- } \\
\text { bus } 21 \text { ) }\end{array}$ & 10 & 28 \\
\hline 30 & $\begin{array}{c}\text { Line } 14 \\
\text { (bus6-bus28) }\end{array}$ & 10 & 29 \\
\hline 31 & $\begin{array}{c}\text { Line } 6 \\
\text { (bus3-bus4) }\end{array}$ & 10 & 30 \\
\hline 32 & $\begin{array}{c}\text { Line } 15 \\
\text { (bus8-bus28) }\end{array}$ & 10 & 31 \\
\hline 33 & Line 41 & 10 & 32 \\
\hline
\end{tabular}

\begin{tabular}{|c|c|c|c|}
\hline & $\begin{array}{c}\text { (bus29- } \\
\text { bus30) }\end{array}$ & & \\
\hline 34 & $\begin{array}{l}\text { Line 26 } \\
\text { (bus14- } \\
\text { bus15) }\end{array}$ & 10 & 33 \\
\hline 35 & $\begin{array}{l}\text { Line } 19 \\
\text { (bus10- } \\
\text { bus17) }\end{array}$ & 10 & 33 \\
\hline 36 & $\begin{array}{c}\text { Line } 3 \\
\text { (bus2-bus4) }\end{array}$ & 10 & 33 \\
\hline 37 & $\begin{array}{c}\text { Line } 2 \\
\text { (bus1-bus3) }\end{array}$ & 10 & 34 \\
\hline 38 & $\begin{array}{c}\text { Line } 1 \\
\text { (bus1-bus2) }\end{array}$ & 10 & 35 \\
\hline 39 & $\begin{array}{l}\text { Line } 35 \\
\text { (bus24- } \\
\text { bus25) }\end{array}$ & 10 & 36 \\
\hline 40 & $\begin{array}{c}\text { Line } 9 \\
\text { (bus5-bus7) }\end{array}$ & 11 & 37 \\
\hline 41 & $\begin{array}{c}\text { Line } 5 \\
\text { (bus2-bus6) }\end{array}$ & 11 & 38 \\
\hline
\end{tabular}

The results for IEEE - 30 bus test system are obtained. The ranking indices are obtained for all the outages. The index which is having highest value indicates the contingency as most severe and the index with lowest value indicates the contingency as less severe.

The results for IEEE - 30 bus test system are obtained. It is seen from the results of exact ranking method that, the line 22 outage has value of ERI as 31. It implies that this is the contingency with more severity and whose outage severely affects the operation of power system hence this contingency is ranked as 1 . Similarly it is seen from the table that, for the line 13 and 24 outages the ERI obtained is 12 which indicates that these are the transmission lines having same severity hence these outages are ranked with identical ranking and so on.

Similarly from the precise ranking method it is seen that, the line 22 outage has a value of PRI as 120.9750 . Hence the line outage 22 is most severe so it is ranked as 1 in the ranking list. Similarly it is seen from the table that, for the line 13 and 24 outages the PRI value is 20.9154 and 20.9496 respectively therefore the line outage 13 is ranked as 7 and line 24 as 6 . This indicates that in the exact ranking indices, where the severity of line 13 and line 24 outages is same, the precise ranking indices distinguish between the two.

Finally the comparison of exact ranking indices (ERI) and precise ranking indices (PRI) based contingency ranking is made in the table. It is seen from the table that, the exact ranking method does not differentiate between the outages with same severity hence label them with identical ranking.

The precise ranking technique addresses the concern of identical ranking for the outages with same severity by taking into account in case there is any line or bus reaching near to its limits following a particular contingency.

Hence precise ranking method distinguishes between the outages having same severity. 


\section{CONCLUSION}

In this paper two methods for the contingency ranking i.e. exact ranking method and precise ranking method were described. Both of these methods correctly identify all the unstable contingencies. Further precise ranking method provides more distinction and information which can enable the power system operator to have more effective control action.

The result shows that the calculation of indices gives a measure of severity of possible line outage contingencies in the system. The highest value of indices provides the severity of outage and has maximum possibility of making the system parameters to go beyond the operating limits.

The effectiveness of this method is demonstrated on IEEE - 14 and IEEE - 30 bus test system respectively. The results clearly have shown that both the methods are effective in ranking the contingencies according to the severity. Also both the methods are compared to know the relative degree of preciseness in ranking the contingencies.

The method used which in addition to voltage variable considers apparent power instead of real power which is more realistic approach. In addition to being accurate and precise, this method is fast enough to be considered for online security analysis in present and future complex mixed power system.

\section{REFERENCES}

[1] Zakir Hussain, Zhe Chen, Paul Thogersen, "Fast and Precise Method of Contingency Ranking in Modern Power System", IEEE conference on applied electrical engineering and computing technologies, (C) 2011 IEEE.

[2] I Musirin, T.K. Abdul Rahaman, "Estimating Maximum Lodability of Weak Bus Identification using FVSI," IEEE power engineering review, (C) 2002 IEEE

[3] I Musirin, T.K. Abdul Rahaman, "Fast Automatic Contingency Analysis and Ranking Technique for Power System Security Assessment", Student conference on Research and Development, 2003 proceedings, Putrajaya Malaysia.

[4] Ozdemir A. and Singh C., "Fuzzy Logic Based MW Contingency Ranking against Masking Problem", in Proc. IEEE Power Eng. Soc. Wint. Meet, jan/Feb. 2001,vol. 2, pp. 504-509.

[5] Hsu Y. Y. and Kuo H. C., "Fuzzy Set Based Contingency Ranking”, IEEE Trans. on Power Systems, vol.7, no.3, Aug. 1992.

[6] Y. Chen and A. Bose, "Direct Ranking for voltage contingency ranking", IEEE Trans. Power Syst., vol. 4, no. 4, pp. 13351344, Nov. 1989.

[7] G.C. Ejebe, H.P. Van Meeteren., B.F. Wollenberg, "Fast contingency screening and evaluation for voltage security analysis", IEEE Trans. Power Syst., vol.3, no.4, pp.1582-1590, Nov. 1988.

[8] Srivastava L., S.N Singh, and J Sharma, "Knowledge Based Neural Network for Voltage Contingency Selection and Ranking", IEEE Proceedings on Generation, Transmission and Distribution, 146(6): 649-656 (Nov. 1999).

[9] Scott Greene, Ian Dobson, Fernando L. Alvarado, Contingency Ranking for Voltage Collapse via Sensitivities from a single nose curve", IEEE Transactions on Power Systems, Vol.14, No.1, February 1999.

[10] Hang Liu, Anjan Bose, and Vaithianathan V., "A Fast Voltage Security Assessment method using Adaptive Bounding", IEEE Transactions on Power Systems Vol.15, No.3, August 2000.

[11] Aydogan Ozedemir, Jae Yun Lim, and Chanan Singh, "Branch Outage Simulation for MVAR Flows: Bounded Network Solution", IEEE Transactions on Power Systems, Vol.18, No.4, November 2003.

[12] C. Subramani, Subhransu Sekhar Das, M Arun Bhaskar, M.Jagdeshkumar, "Simulation Technique for Voltage Stability Analysis and Contingency Ranking in Power Systems",
Electronics Engineering 2010.

[13] M.A. Kamarposhti, H.Lesani, "Contingency Analysis for Voltage Stability Analysis using Continuation Power Flow Method", International Journal of Resent Trends in Electrical and Electronics Engineering ISSSN-1392-1215, 2010.

[14] Shobha Shankar, T.Ananthapadmanabha, "Fuzzy Approach to Critical Bus Ranking Under Normal and Line Outage Contingencies", International Journal on Soft Computing Vol.2, No.1, pp 59-69, Feb 2011.

[15] Ahamadi Kamarposhti, Barak Mozafari "Study the Effects of Power Plant Outages on Maximum Loading in Power System", Journal of Basic and Applied Scientific Research, pp.24102416, 2011.

[16] Veenavati Jagadishprasad Mishra, Manisha D. Khardenvis, "Contingency Analysis of Power System", International Conference on Emerging Frontiers in Technology for Rural Area, 2012.

[17] J.B.Gupta, "A Course in Power Systems", $X^{\text {th }}$ Edition.

[18] D.P.Kothari, I.J.Nagrath, "Modern Power System Analysis", IV $^{\text {th }}$ Edition.

[19] K.Uma Rao, "Computer Techniques And Models in Power Systems", I.K.International I ${ }^{\text {st }}$ Edition.

[20] Allen J. Wood, Bruce F. Wollenberg, "Power Generation Operation and Control", Second Edition.

[21] Hadi Saadat, "Power System Analysis", Tata-Mcgraw Hill Publication, First edition. 